these patients who have a high risk of hepatic decompensation or death within 12 months, the rate of death was $7.8 \%$ during treatment and the 12 weeks posttreatment. These deaths and most safety events were associated with advanced liver disease and not considered treatment related. The largest phase III studies (COSMOS, ION1 and ION3, SAPPHIRE) did not report any death during DAA treatment and severe adverse events occurring during therapy were considered to be unlikely related to DAAs. ${ }^{3}$ In reallife clinical settings, mortality has been observed in around $0.5 \%(0.3 \%$ for the French ANRS CO22 HEPATHER cohort and $0.6 \%$ for the US TARGET cohort).

In our tertiary care centre (part of the CO22 HEPATHER cohort), we have treated 996 patients, including 400 (40\%) with cirrhosis, with a DDA-based regimen and $91.5 \%$ of patients achieved a sustained virological response (SVR12).

Twelve deaths (1.2\%) occurred during the treatment or the 12 -week posttreatment period (table 1), a prevalence lower than the $2.2 \%$ mortality reported in the Cupic study targeting only a cirrhotic population treated by pegylated interferon, ribavirin and a first-generation protease inhibitor. The median age at death was 68 (IQR, 63-71) years; seven patients $(58 \%)$ were males, eight $(66 \%)$ had cirrhosis, including six with a Child-Pugh score B or C. Six deaths (50\%) occurred under DAA treatment and six during the follow-up. Four patients (33\%) died from a liver-related event (one with hepatocellular carcinoma (HCC) and three with end-stage liver disease (ESLD)). In one of them, with a Child-Pugh score B, ESLD and consequent death were assumed to be likely related to the 6 weeks of ombitasvir+ritonavir + paritaprevir + ribavirin.

We now know that protease inhibitors are contraindicated in decompensated cirrhosis. In three patients $(25 \%)$ death was related to sepsis and in two (17\%) patients death was related to haemorrhagic stroke, the risk of which is 2.5 -fold higher in $\mathrm{HCV}$ infection with significant liver fibrosis. Finally, three $(25 \%)$ patients died of sudden death at weeks 5, 8 and 12 under sofosbuvir-based treatment: one had a

\section{Mortality associated with the treatment of HCV with direct-acting antivirals}

We read with interest the study by Welzel et $a l^{1}$ confirming the high efficacy of oral direct-acting antiviral agents (DAAs) for the treatment of chronic HCV infection. ${ }^{2}$ Results about safety are less clear, since in Child-Pugh score A cirrhosis with unbalanced diabetes and active smoking; one was a kidney allograft recipient with fibrosis F3 who was treated by amiodarone and had a pacemaker; the third patient had a F2 fibrosis and had a prior history of ischaemic heart disease, atrial fibrillation and a pacemaker.

Our data suggest that DAA-associated mortality is higher in real-life clinical 
settings than in phase III clinical trials, and can be observed with any available DAA combination. One-third of mortality cases were related to sudden deaths in patients with elevated cardiac risk, including one amiodarone-treated patient. We have previously reported the risk of cardiac arrythmia in patients treated by sofosbuvir, with causality suggested by the timing of occurrence, the relapse on reintroduction of sofosbuvir and the pacemaker data. ${ }^{4}$ The preclinical toxicology studies described heart degeneration and inflammation with over dosage of the main metabolite of sofosbuvir (GS-331007), and another NS5B HCV nucleotide polymerase inhibitor, BMS-986094, has been withdrawn due to an association with cardiomyopathy. This risk is not only restricted to amiodaronetreated patients ${ }^{4}$ and particular attention should be applied when extending DAAs to any infected patient, especially in those with cardiac risks.

Anne Laurain, Laura Kramer, Philippe Sultanik, Anaïs Vallet-Pichard, Philippe Sogni,

Stanislas Pol

Université Paris Descartes; APHP, Unité d'Hépatologie, Hôpital Cochin; INSERM U1223 and USM20, Institut Pasteur, Paris, France

Correspondence to Dr Anne Laurain, Département d'Hépatologie, Hôpital Cochin, 27 rue du Faubourg Saint Jacques, 75679 Paris Cedex 14, France; anne.laurain@aphp.fr

Acknowledgements Thanks to Darragh Duffy for the revision of the manuscript.

Contributors AL and LK have equally contributed. Conception and design: AL, LK and SP. Analysis and interpretation of the data: AL, LK and SP. Drafting of the article: AL, LK and SP. Critical revision of the article for important intellectual content: all the authors. Final approval of the article: all the authors.

Competing interests $L K$ is a board member of Gilead. AV-P received personal fees from Gilead, BMS, Janssen, MSD, Abbvie, Roche. PS was board member of Gilead and BMS. Workshop or meeting invitation: Gilead, BMS, MSD, AbbVie, Mayoloy-Spindler, Janssen. SP was speaker for BMS, Boehringer Ingelheim, Janssen Gilead, Roche, MSD, Novartis, Abbvie; received grants from BMS, Gilead, Roche, MSD and is board member of BMS, Boehringer Ingelheim, Janssen, Gilead, Roche, MSD, Abbvie.

Provenance and peer review Not commissioned; internally peer reviewed.

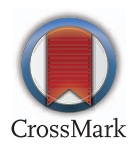

To cite Laurain A, Kramer L, Sultanik P, et al. Gut 2018;67:197-198.

Revised 29 January 2017

Revised 27 February 2017

Accepted 28 February 2017

Published Online First 23 March 2017

Gut 2018;67:197-198.

doi:10.1136/gutjnl-2017-313869

\section{REFERENCES}

1 Welzel TM, Petersen J, Herzer K, et al. Daclatasvir plus sofosbuvir, with or without ribavirin, achieved high sustained virological response rates in patients with $\mathrm{HCV}$ infection and advanced liver disease in a real-world cohort. Gut 2016:65:1861-70.

2 Pol S, Bourliere M, Lucier S, et al. Safety and efficacy of daclatasvir-sofosbuvir in HCV genotype 1-mono-infected patients. J Hepatol 2017;66:39-47.

3 Muir A J, Pang PS, Stamm LM. et al. Detecting drug-induced liver injury in patients with decompensated chronic hepatitis C: a review of the SOLAR-1 and SOLAR-2 studies. Hepatology (Baltimore, MD) 2015;62:1(SUPPL) 96A. http://dx. doi.org/10.1002/hep.27898

4 Fontaine $\mathrm{H}$, Lazarus A, Pol S, et al. Bradyarrhythmias associated with Sofosbuvir treatment. N Engl J Med 2015;373:1886-8. 